\title{
Statistical Probes of Reionization with 21 Centimeter Tomography
}

\section{Citation}

Furlanetto, Steven R., Matias Zaldarriaga, and Lars Hernquist. 2004. "Statistical Probes of Reionization with 21 Centimeter Tomography." The Astrophysical Journal 613 (1): 16-22. https:// doi.org/10.1086/423028.

\section{Permanent link}

http://nrs.harvard.edu/urn-3:HUL.InstRepos:41381831

\section{Terms of Use}

This article was downloaded from Harvard University's DASH repository, and is made available under the terms and conditions applicable to Other Posted Material, as set forth at http:// nrs.harvard.edu/urn-3:HUL.InstRepos:dash.current.terms-of-use\#LAA

\section{Share Your Story}

The Harvard community has made this article openly available.

Please share how this access benefits you. Submit a story.

Accessibility 
DRAFT VERSION JUNE 8, 2018

Preprint typeset using $\mathrm{LT}_{\mathrm{E}} \mathrm{X}$ style emulateapj v. 9/08/03

\title{
STATISTICAL PROBES OF REIONIZATION WITH 21 CM TOMOGRAPHY
}

\author{
STEVEn R. FurlanetTo ${ }^{1}$, MATIAS ZALDARRIAGA ${ }^{2,3}$, \& LARS HERNQUist ${ }^{2}$ \\ Draft version June 8, 2018
}

\begin{abstract}
We consider the degree to which " $21 \mathrm{~cm}$ tomography" of the high-redshift Universe can distinguish different ionization histories. Using a new analytic model for the size distribution of H II regions that associates these ionized bubbles with large-scale galaxy overdensities, we compute the angular power spectrum and other statistical properties of the $21 \mathrm{~cm}$ brightness temperature during reionization. We show that the $\mathrm{H}$ II regions imprint features on the power spectrum that allow us to separate histories with discrete bubbles from those with partial uniform ionization (by, for example, X-rays). We also show that "double reionization" scenarios will modify the morphology of the bubbles in ways that depend on the mechanism through which the first generation of sources shuts off. If, for example, the transition occurs globally at a fixed redshift, the first generation imprints a persistent feature on the $21 \mathrm{~cm}$ power spectrum. Finally, we compare our model to one in which voids are ionized first. While the power spectra of these two models are qualitatively similar, we show that the underlying distributions of neutral hydrogen differ dramatically and suggest that other statistical tests can distinguish them. The next generation of low-frequency radio telescopes will have the sensitivity to distinguish all of these models and strongly constrain the history and morphology of reionization.
\end{abstract}

Subject headings: cosmology: theory - intergalactic medium - diffuse radiation

\section{INTRODUCTION}

Recently, a great deal of effort - both observational and theoretical - has been focused on understanding the reionization of hydrogen in the intergalactic medium (IGM) at $z \gtrsim 6$. Several independent observational techniques offer complementary views of this landmark event. The most straightforward method is to seek extended troughs of complete Ly $\alpha$ absorption in the spectra of high-redshift quasars. Evidence for this Gunn \& Peterson (1965) effect has been found near $z \sim 6.5$ (Becker et al. 2001; White et al. 2003). Unfortunately, the optical depth of a fully neutral medium is so high that current measurements only require a mean neutral fraction $\bar{x}_{H} \geq 10^{-3}$, and even this limit has been disputed (Songaila 2004). Studies of the rapidly growing ionizing background (Fan et al. 2002) and the Strömgren spheres surrounding the quasars (Wvithe \& Loeb 2004) suggest larger neutral fractions but depend on detailed modeling. A second constraint arises because free electrons scatter cosmic microwave background (CMB) photons, washing out intrinsic anisotropies and generating a polarization signal (Zaldarriaga 1997). This provides an integral constraint on the reionization history; recent measurements of the CMB imply that $\bar{x}_{H}$ must have been small at $z \gtrsim 14$ (Kogut et al. 2003; Spergel et al. 2003). Finally, the relatively high temperature of the $\operatorname{Ly} \alpha$ forest at $z \sim 2-4$ suggests an order unity change in $\bar{x}_{H}$ at $z \lesssim 10$ (Theuns et al. 2002; Hui \& Haiman 2003), although this argument depends on the characteristics of He II reionization (e.g., Sokasian et al. 2002).

Taken together, these three sets of observations suggest a complex ionization history extending over a large redshift interval $(\Delta z \sim 10)$. This is inconsistent with a generic picture of fast reionization (e.g., Barkana \& Loeb 2001, and refer-

1 Division of Physics, Mathematics, \& Astronomy; California Institute of Technology; Mail Code 130-33; Pasadena, CA 91125; sfurlane@tapir.caltech.edu

${ }^{2}$ Harvard-Smithsonian Center for Astrophysics, 60 Garden St., Cambridge, MA 02138

3 Jefferson Laboratory of Physics, Harvard University, Cambridge, MA 02138 ences therein). The results seem to indicate strong evolution in the sources responsible for reionization, and a precise measurement of the ionization history would strongly constrain early structure formation (Wvithe \& Loeb 2003; Cen 2003; Haiman \& Holder 2003; Sokasian et al. 2003b). Unfortunately, extracting such detailed information requires new observational techniques. One of the most exciting possibilities is "21 cm tomography" of the high-redshift IGM (Scott \& Rees 1990; Kumar et al. 1995; Madau et al. 1997), in which one maps the distribution of neutral hydrogen on large scales through its hyperfine transition. By probing a specific spectral line, $21 \mathrm{~cm}$ surveys measure the time history of reionization, and unlike the $\operatorname{Ly} \alpha$ forest they do not suffer from saturation problems. In fact, the signal is sufficiently weak that foregrounds from the Galaxy and a wide variety of extragalactic objects pose substantial challenges to these experiments (Oh \& Mack 2003; Di Matteo et al. 2004). Fortunately, the known foregrounds all have smooth continuum spectra, which should allow frequency cleaning to high accuracy because the $21 \mathrm{~cm}$ signal has spectral structure on small scales (Zaldarriaga et al. 2004, hereafter ZFH04; Morales \& Hewitt 2003; Cooray \& Furlanetto 2004).

However, predicting the $21 \mathrm{~cm}$ signals from reionization has proven difficult. One method is to use numerical simulations of reionization (Ciardi \& Madau 2003; Furlanetto et al. 2004a; Gnedin \& Shaver 2003), but computational costs have so far limited the simulations to subtend (at best) a few resolution elements of the $21 \mathrm{~cm}$ maps. Analytic models of reionization can extend to larger scales but require some way to describe the complexities of structure formation and radiative transfer. The simplest approach, in which we assign each galaxy its own H II region, does a poor job of matching the large ionized bubbles found in simulations (Ciardi et al. 2003; Sokasian et al. 2003a). We have recently developed a model that associates $\mathrm{H}$ II regions with large-scale fluctuations in the density field and reproduces the major features seen in simulations (Furlanetto et al. 2004b, hereafter Paper I). This allows us, for the first time, to make quantitative predictions about the $21 \mathrm{~cm}$ signal at reionization. With high signal-to- 
noise $21 \mathrm{~cm}$ maps, we can directly measure the distribution of $\mathrm{H}$ II regions in order to test our model. However, the signals are sufficiently weak that such maps will be difficult to make; fortunately, statistical measurements of the data also contain a great deal of information about reionization (ZFH04). In this paper, we use the model of Paper I to predict the $21 \mathrm{~cm}$ angular power spectrum for several reionization scenarios, with an emphasis on how the measurements help distinguish the crucial features of these different pictures. In $\$ 2$ we briefly review our model for reionization. We then show how $21 \mathrm{~cm}$ measurements can distinguish different reionization histories in $\$ 3$ and how they can distinguish different models of reionization in $\$ 4$ We conclude in $\$ 5$

In our numerical calculations, we assume a $\Lambda$ CDM cosmology with $\Omega_{m}=0.3, \Omega_{\Lambda}=0.7, \Omega_{b}=0.046, H=$ $100 h \mathrm{~km} \mathrm{~s}^{-1} \mathrm{Mpc}^{-1}$ (with $h=0.7$ ), $n=1$, and $\sigma_{8}=0.9$, consistent with the most recent measurements (Spergel et al. 2003).

\section{A MODEL FOR REIONIZATION}

Recent numerical simulations (e.g., Sokasian et al. 2003a) show that reionization proceeds "inside-out" from high density clusters of sources to voids, at least when the sources resemble star-forming galaxies (e.g., Springel \& Hernquist 2003). We therefore associate H II regions with large-scale overdensities. We assume that a galaxy of mass $m_{\text {gal }}$ can ionize a mass $\zeta m_{\text {gal }}$, where $\zeta$ is a constant that depends on the efficiency of ionizing photon production, the escape fraction, the star formation efficiency, and the number of recombinations. Values of $\zeta \sim 10-40$ are reasonable for normal star formation, but very massive stars can increase the efficiency by an order of magnitude (Bromm et al. 2001b). The criterion for a region to be ionized by the galaxies contained inside it is then $f_{\text {coll }}>\zeta^{-1}$, where $f_{\text {coll }}$ is the fraction of mass bound to halos above some $m_{\min }$. We will normally assume that this minimum mass corresponds to a virial temperature of $10^{4} \mathrm{~K}$, at which point hydrogen line cooling becomes efficient. In the extended Press-Schechter model (Lacey \& Cole 1993), this places a condition on the mean overdensity within a region of mass $m$,

$$
\delta_{m} \geq \delta_{x}(m, z) \equiv \delta_{c}(z)-\sqrt{2} K(\zeta)\left[\sigma_{\min }^{2}-\sigma^{2}(m)\right]^{1 / 2},
$$

where $K(\zeta)=\operatorname{erf}^{-1}\left(1-\zeta^{-1}\right), \sigma^{2}(m)$ is the variance of density fluctuations on the scale $m, \sigma_{\min }^{2}=\sigma^{2}\left(m_{\min }\right)$ and $\delta_{c}(z)$ is the critical density for collapse.

Paper I showed how to construct the mass function of $\mathrm{H}$ II regions from $\delta_{x}$ in an analogous way to the halo mass function (Press \& Schechter 1974; Bond et al. 1991). The barrier in equation (1) is well approximated by a linear function, $\delta_{x} \approx$ $B(m, z)=B_{0}+B_{1} \sigma^{2}(m)$. In that case the mass function has an analytic expression (Sheth 1998):

$$
m \frac{d n}{d m}=\sqrt{\frac{2}{\pi}} \frac{\bar{\rho}}{m}\left|\frac{d \ln \sigma}{d \ln m}\right| \frac{B_{0}}{\sigma(m)} \exp \left[-\frac{B^{2}(m, z)}{2 \sigma^{2}(m)}\right],
$$

where $\bar{\rho}$ is the mean density of the universe. Equation (2) gives the comoving number density of $\mathrm{H}$ II regions with masses in the range $(m, m+d m)$. The crucial difference between this formula and the standard Press-Schechter mass function arises from the fact that $\delta_{x}$ is a (decreasing) function of $m$. The barrier is more difficult to cross as one goes to smaller scales, which gives the bubbles a characteristic size. In contrast, the barrier used in constructing the halo mass function is independent of mass, yielding the usual power law behavior at small masses. The characteristic scale depends primarily on $\bar{x}_{H}$.
Paper I also showed how to construct the power spectrum of the quantity $\psi=x_{H}(1+\delta)$. We explicitly computed the correlation function of the $\mathrm{H}$ II regions $\xi_{x x}$ in terms of the mass function in equation (2), and we computed the density correlation function $\xi_{\delta \delta}$ and cross correlation between density and ionization fraction $\xi_{x \delta}$ using the halo model (e.g., Cooray \& Sheth 2002). We combined these correlation functions to get $\xi_{\psi}$, the correlation function of $\psi$,

$$
\xi_{\psi}=\xi_{x x}\left(1+\xi_{\delta \delta}\right)+\bar{x}_{H}^{2} \xi_{\delta \delta}+\xi_{x \delta}\left(2 \bar{x}_{H}+\xi_{x \delta}\right) .
$$

ZFH04 showed how to convert this to the three-dimensional power spectrum $P_{\psi}$ and then to the (observable) angular power spectrum of the $21 \mathrm{~cm}$ brightness temperature $\delta T_{b}$, essentially by doing a Fourier transform. Here

$$
\begin{aligned}
\delta T_{b} \approx 23 \psi & \times\left(\frac{T_{S}-T_{\mathrm{CMB}}}{T_{S}}\right)\left(\frac{\Omega_{b} h^{2}}{0.02}\right) \\
& \times\left[\left(\frac{0.15}{\Omega_{m} h^{2}}\right)\left(\frac{1+z}{10}\right)\right]^{1 / 2} \mathrm{mK},
\end{aligned}
$$

with $T_{S}$ the spin temperature of the gas. In all of the calculations here, we assume $T_{S} \gg T_{\mathrm{CMB}}$, which should be reasonable even relatively early in reionization (see $\$ 2$ of ZFH04). We further assume perfect frequency resolution in computing the angular power spectrum; our results therefore do not depend on the experimental setup. The effects of finite bandwidth are described in ZFH04 and Paper I. We refer the reader to these papers for more details on our approach. Note that these methods to construct the angular power spectrum of $\delta T_{b}$ can be applied to any model of the H II regions.

\section{COMPLEX REIONIZATION}

Paper I examined the power spectra of standard reionization scenarios in which all the ionizations are confined to discrete $\mathrm{H}$ II regions. We showed that the power spectrum evolves rapidly throughout reionization, allowing one to reconstruct the ionization history in such a simple scenario. Here we extend our model in order to consider the effects of more complicated reionization processes.

\subsection{A Partially Ionized IGM}

We first consider how well $21 \mathrm{~cm}$ observations can distinguish a model with all ionizations inside of $\mathrm{H}$ II regions from one with a uniform component, such as may happen through ionization by X-rays (Oh 2001; Venkatesan et al. 2001; Ricotti \& Ostriker 2003; Madau et al.|2003) or decaying particles (Sciama 1982; Hansen \& Haiman 2004). Suppose that the IGM has a uniform ionization fraction $\bar{x}_{u}(z)$. On top of this uniform level are some variations in the neutral fraction owing to isolated H II regions. In this case, the condition $f_{\text {coll }}>\zeta^{-1}\left(1-\bar{x}_{u}\right)$ replaces the barrier in our model: each galaxy can produce a larger ionized bubble with the same number of ionizing photons. However, rather than varying from zero to unity, we have $0<x_{H}<\left(1-\bar{x}_{u}\right)$. This damps the fluctuations from the bubbles, requiring the replacements $\xi_{x x} \rightarrow\left(1-\bar{x}_{u}\right)^{2} \xi_{x x}$ and $\xi_{x \delta} \rightarrow\left(1-\bar{x}_{u}\right) \xi_{x \delta}$. Otherwise our formalism is unchanged.

Figure 1 contrasts such a case with a "standard" history. The thin curves assume $\zeta=40$ and $\bar{x}_{u}=0$, while the thick curves have $\zeta=12$ and set $\bar{x}_{u}(z)$ to force the total neutral fraction to match in the two cases. The uniform component is thus responsible for $70 \%$ of the ionizations. When $\bar{x}_{H} \approx 1$, the two curves are nearly identical because the bubble feature is intrinsically weak. However, while the bubbles rapidly imprint 


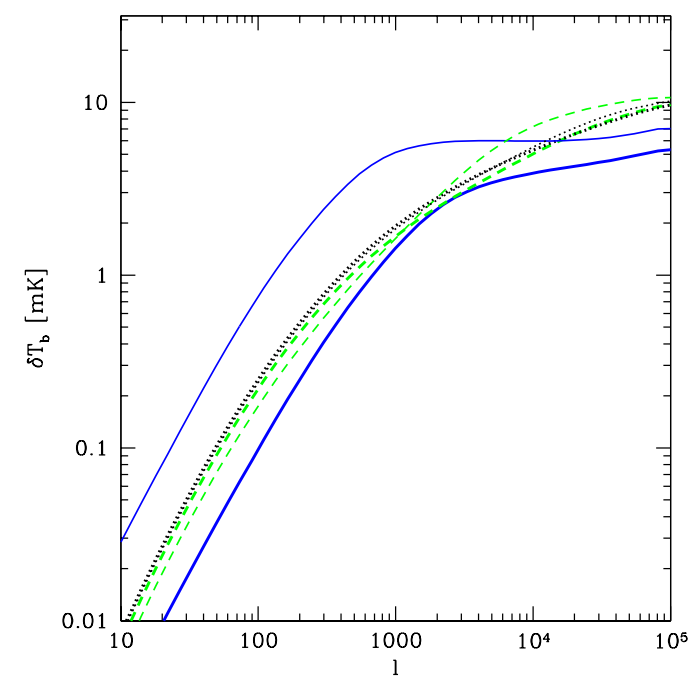

FIG. 1.- The $21 \mathrm{~cm}$ fluctuation amplitude as a function of redshift. The thick curves have $\zeta=12$ plus a uniformly ionized component, while the thin curves have $\zeta=40$. The ionization fraction $\bar{x}_{H}(z)$ is identical in the two models. The curves have: $\bar{x}_{H}=0.96$ (dotted), $\bar{x}_{H}=0.8$ (dashed) and $\bar{x}_{H}=0.26$ (solid).

a feature on the power spectrum if $\bar{x}_{u}=0$, uniform ionization strongly damps the power from the $\mathrm{H}$ II regions. As a result, in such a scenario the $21 \mathrm{~cm}$ fluctuations trace those of the density (normalized by $1-\bar{x}_{u}$ ), at least until reionization is almost complete. Consequently, the amplitude of $\delta T_{b}$ is suppressed by $25-50 \%$ on scales near the characteristic bubble size, even when $\bar{x}_{H}$ is large. We find that, unless reionization is near completion, the bubbles need to provide a majority of the ionizations in order for them to have an appreciable effect on the power spectrum. This provides a clear test for whether Strömgren spheres from ultraviolet photons or smoother ionization from some other source dominate the morphology of reionization, even if the survey is unable to make high signalto-noise maps.

\subsection{Double Reionization}

Recently, a number of theoretical models for reionization have attempted to reconcile the CMB and quasar data by postulating an early generation of sources with high ionizing efficiency (most often because they contain massive, metal free stars) along with a self-regulation mechanism that switches to normal star formation with a lower ionizing efficiency (Wvithe \& Loeb 2003; Cen 2003; Haiman \& Holder 2003; Sokasian et al. 2003b). Such scenarios can lead to a plateau in the ionized fraction or even "double" reionization, in which ionized phases bracket a mostly neutral period. In this section, we explore the signatures such scenarios imprint on the $21 \mathrm{~cm}$ power spectrum through some simple toy models.

We consider an early generation of sources with $\left(\zeta_{1}, m_{\min , 1}\right)$ and a later one with $\left(\zeta_{2}, m_{\min , 2}\right)$. In the initial phase only the first generation is important, so the ionization simply follows the model of Paper I. We then end this generation according to one of two conditions. The first choice shuts down these sources once the universe reaches some uniform level of ionization at redshift $z_{1}$. For example, the early phase could be caused by X-rays or decaying particles, as described in $\$ 3.1$ A better-motivated scenario could be the following. Suppose that the first generation consisted of massive, metal-free stars; the natural self-regulation condition halts the formation of these stars when the metallicity in collapsed objects passes some threshold (Bromm et al. 2001a; Mackev et al. 2003; Yoshida et al. 2003). These sources will stop forming soon after they first appear in any given region (or in other words soon after the region is ionized for the first time) but they will continue to form in the remaining neutral regions. Thus the first generation will persist until the entire universe has been ionized. Our toy model makes the simplification that the first generation was able to keep the universe ionized until the transition occurs; we implicitly neglect recombinations within regions that were ionized before $z_{1}$.

While the second generation is still rare, recombination in the IGM will be the dominant effect. Such an era is obviously easy to identify with $21 \mathrm{~cm}$ tomography because it is accompanied by a corresponding increase in $\bar{x}_{H}$ and hence in the signal (Furlanetto et al. 2004a). If the recombination rate were uniform throughout the IGM, we would have the power spectrum $P_{\psi}=\bar{x}_{H}^{2} P_{\delta \delta}$. Because the recombination rate is a function of the density, we also get fluctuations in the neutral fraction that amplify $\delta T_{b}$. In the limit that the elapsed time is smaller than the recombination time, we would have $P_{x x} \approx\left[1-\bar{x}_{i}(z) / \bar{x}_{i}\left(z_{1}\right)\right]^{2} P_{\delta \delta}$, where $\bar{x}_{i}$ is the mean ionized fraction. Unfortunately, $x_{H}(\delta, z)$ from recombinations is a nonlinear function, so the detailed evolution must await numerical simulations. Given the weak large-scale fluctuations present at high-redshifts, we do not expect recombinations to amplify the power by more than a few tens of percent, and it should not imprint prominent features into the power spectrum. (Note that allowing extra recombinations in regions that were ionized before $z_{1}$ would increase this amplification factor.) In our formalism, we can implement this scenario using the results of $\$ 3.1$ with the extra wrinkle that $\bar{x}_{u}$ can decrease with cosmic time.

Another prescription shuts off the first generation before those sources can fully ionize the universe. At $z_{1}$, the universe has developed a patchwork of $\mathrm{H}$ II regions but they have not yet overlapped completely. This choice is appropriate if the self-regulation mechanism is not local. For example, the buildup of an ultraviolet background in the Lyman-Werner bands could halt $\mathrm{H}_{2}$ cooling and shut off star formation in small halos (Haiman et al. 1997), or an X-ray background could heat the universe and raise the Jeans mass. In this model, the first generation imprints a set of ionized bubbles, within which most of the second generation sources grow (because both appear in the same overdense regions). The bubbles grow only slowly until the total number of ionizations from the second generation become comparable to that of the first; after this point the evolution approaches the normal behavior. If we neglect recombinations within $\mathrm{H}$ II regions, the barrier can be set up as follows. Consider a region of mass $m$ at $z_{2}<z_{1}$; as in Paper I we need the condition for sources inside this region to ionize it. The number of ionizations is simply the sum of those from both generations. Thus we find that the excursion set barrier $\delta_{x}(m)$ will be the solution of

$$
\begin{aligned}
1= & \left(\zeta_{1}-\zeta_{2}\right) \operatorname{erfc}\left\{\frac{\delta_{c}\left(z_{1}\right)-\delta_{x}(m)}{\sqrt{2\left[\sigma^{2}\left(m_{\min , 1}\right)-\sigma^{2}(m)\right]}}\right\} \\
& +\zeta_{2} \operatorname{erfc}\left\{\frac{\delta_{c}\left(z_{2}\right)-\delta_{x}(m)}{\sqrt{2\left[\sigma^{2}\left(m_{\min , 2}\right)-\sigma^{2}(m)\right]}}\right\} .
\end{aligned}
$$

Here the complementary error functions are the fraction of collapsed gas above the mass thresholds at the two redshifts. 


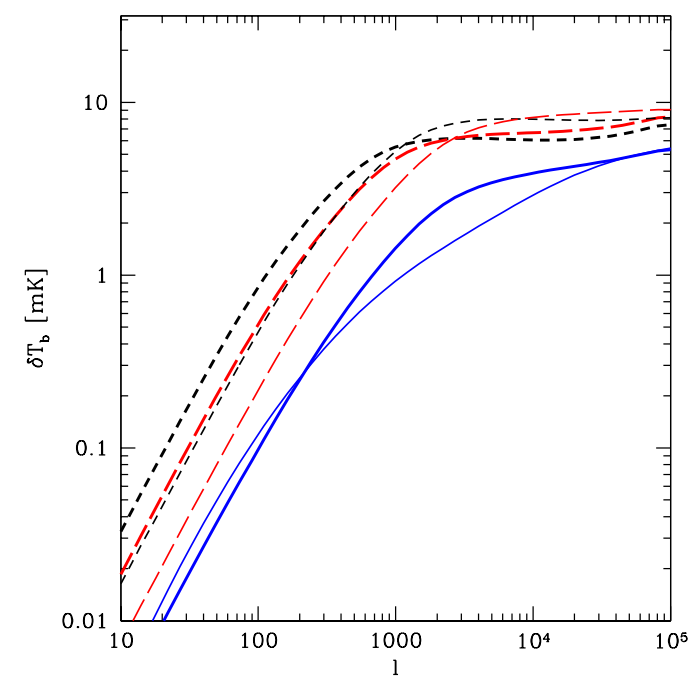

FIG. 2.- The $21 \mathrm{~cm}$ power spectrum in three different models of "double reionization." The solid curves assume that the universe has $\bar{x}_{u}=0.5$, the long-dashed curves assume $z_{1}=18$ with $\zeta_{1}=500$, and the short-dashed curves assume $z_{1}=18$ with $\zeta_{1}=105$ and $m_{\min }$ ten times smaller than our default value. In all cases, $\zeta_{2}=12$. The thin curves are for $z=16\left(\bar{x}_{H}=0.54\right)$ and the thick curves are for $z=12\left(\bar{x}_{H}=0.73\right)$.

This is a more complicated expression than in Paper I, but $\delta_{x}$ is still approximately linear in $\sigma^{2}$. With the new barrier, the formalism from Paper I carries over without further modification. We could crudely incorporate recombinations into this model by decreasing $\zeta_{1}$ as redshift decreases. This is not exactly correct, because it would cause the bubbles to shrink rather than recombining at all radii. However, this may not be a serious problem because, according to our prescription, both types of sources will primarily form within the same highlybiased regions. Even a relatively small number of second generation ionizing sources could be enough to halt recombinations within the bubble unless the recombination time is much smaller than the Hubble time. In any case, the main feature of this model is that the first generation bubbles imprint a particular scale on the power spectrum. If recombination is significant (but not complete) and the second generation sources reside in partially-ionized bubbles, their own H II regions will grow faster than normal and will quickly reach this scale, at which point their expansion will slow. If, on the other hand, recombination is nearly complete in these regions, all evidence of the first generation disappears and the single generation description of Paper I would apply.

Figure 2 contrasts the power spectra of three scenarios for double reionization. In the solid curves, the first sources uniformly ionize the IGM to $\bar{x}_{u}=0.5$ (or, if we neglect amplification due to recombinations, the IGM has recombined to this point after a phase of full ionization). In the other models, we end the first generation at $z_{1}=18$ when $\bar{x}_{H}=0.5$; the dotted and dashed curves correspond to two different sets of parameters for the first generation of sources. In all cases the second generation has $\zeta_{2}=12$ and ionizes $\sim 4 \%(z=16)$ and $\sim 23 \%(z=12)$ of the IGM. Note that we have ignored recombinations after $z_{1}$ in order to isolate the effects of different prescriptions. The most obvious feature of the second type of transition is that the bubble size remains approximately constant with redshift. This contrasts sharply with scenarios in which a single set of sources is responsible for reionization, for which the bubble scale changes rapidly over $\Delta z \sim 1$ (Paper I). We suggest that this plateau in the bubble scale is a clear indication that the transition between generations occurs rapidly and in a non-local manner. It is, however, difficult to distinguish between the two scenarios containing relic bubbles. As described in Paper I, this is because the scale of the H II regions depends only on the behavior of the large scale density fluctuations and is fixed almost entirely by $\bar{x}_{H}$. In the uniform model, the amplitude of $\delta T_{b}$ is significantly smaller and the bubble features are suppressed, just like in \$3.1 Note also that in this case the bubble feature grows rapidly, because the $\mathrm{H}$ II regions expand into a pre-ionized medium. This stands in stark contrast to the dashed curves. While including recombination could increase the overall amplitude of the uniform model by a small amount, it would not affect the bubble feature.

\section{OUTSIDE-IN REIONIZATION}

In Paper I and in $\$ 3$ we have examined how the parameters of the ionizing sources change the $21 \mathrm{~cm}$ power spectrum; however, we have always worked within the model described in $\$ 2$ Many other models for reionization exist, and we now consider whether the $21 \mathrm{~cm}$ signal can discriminate between them. The most popular model assumes that the recombination rate controls ionization (Miralda-Escudé et al. 2000). Thus gas elements below a fixed density threshold are ionized first, with the threshold increasing as reionization progresses. We refer to this as "outside-in" reionization because voids are ionized before the dense regions, where galaxies sit. A simple way to describe such a process is to assume that regions below a fixed mean overdensity $\delta_{v}<0$ are ionized. We can then construct the mass function of the ionized bubbles through the excursion set formalism; the result is identical to the Press \& Schechter (1974) halo mass function except that $\left|\delta_{v}\right|$ replaces the collapse threshold. ${ }^{4}$ We fix $\delta_{v}$ by requiring the fraction of mass in ionized regions with $m>\zeta m_{\min }$ (i.e., larger than an $\mathrm{H}$ II region around the smallest allowed galaxy) to equal $\zeta f_{\text {coll }}$. This prescription is undoubtedly an oversimplification of the Miralda-Escudé et al. (2000) picture, because it completely neglects the source locations, the cosmic web, etc. But it nonetheless serves to illustrate the importance of our basic assumptions. The only additional ingredient we need to construct the power spectrum is the bias of ionized regions. With the above prescription, we have

$$
b_{v}(m, z)=-\left(1+\frac{\delta_{v}^{2} / \sigma^{2}-1}{\left|\delta_{v}\right|}\right) .
$$

This is the same expression as for the halo bias in the PressSchechter model (Mo \& White 1996), except that the bias is negative because the number density of voids increases in underdense regions. ${ }^{5}$

We compare the outside-in angular power spectrum to the inside-out model of Paper I in Figure 3 We have normalized the two models to have identical $\bar{x}_{H}(z)$. We see that, until relatively late in reionization, the fluctuation amplitude and the characteristic bubble scale are similar in the two models. This

4 In principle, we should include the "void-in-cloud" process described by Sheth \& van Weygaert (2003) in order to remove those voids that have been consumed by collapsed objects. However, the regime in which we are interested has $\left|\delta_{v}\right| \ll \delta_{c}$, so this process turns out to have no effect on our results.

${ }^{5}$ With this prescription, we can have the cross-correlation between a halo and a void $\xi_{h v}<-1$, which is unphysical. In these situations we set $\xi_{h v}=-1$; fortunately, this has a negligible effect on our results. 


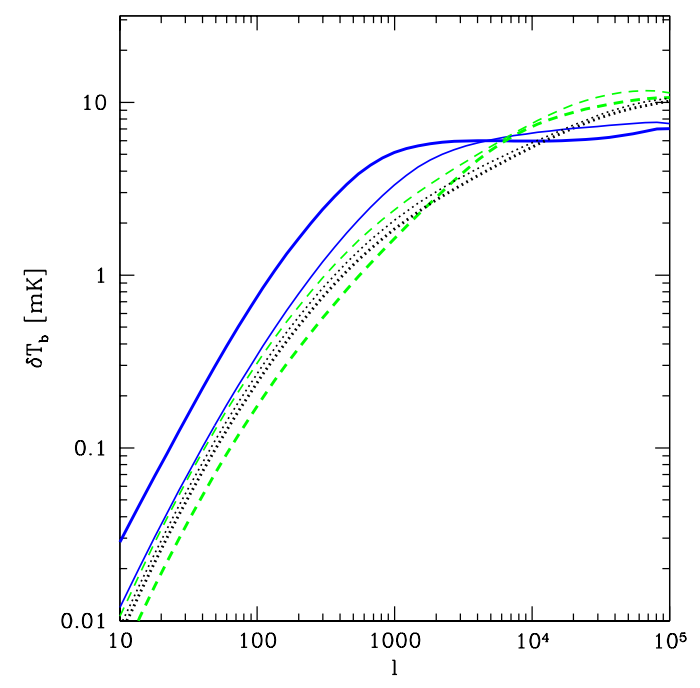

FIG. 3. - The $21 \mathrm{~cm}$ power spectrum in the inside-out model of Paper I (thick lines) and the outside-in model in which voids are ionized first (thin lines). In both cases, $\zeta=40$. The curves are: $\bar{x}_{H}=0.96(z=18$, dotted $)$, $\bar{x}_{H}=0.8(z=15$, dashed $)$, and $\bar{x}_{H}=0.26(z=12$, solid $)$.

is because both models associate $\mathrm{H}$ II regions with large-scale fluctuations in the density field. The only difference is that the void model barrier $\delta_{v}$ is independent of scale but $\delta_{x}$ increases nearly linearly with $\sigma^{2}$ in the inside-out model. As a result, the void model has somewhat more ionized gas in smaller regions and a somewhat smaller characteristic scale; however, these differences may not be robust given the approximate methods used to construct the size distribution. On the other hand, the similarity shows explicitly that, regardless of the detailed model, the characteristic bubble size is large (and in the ideal range for $21 \mathrm{~cm}$ tomography) so long as the sizes of $\mathrm{H}$ II regions are determined by fluctuations in the large-scale density field. A more fundamental difference between the two models is that the void model has significantly more large-scale power, at least until shot noise from the bubbles begins to dominate at $z \sim 13 .^{6}$ By associating ionized regions with underdensities, the void model amplifies large-scale power. In contrast, inside-out reionization washes out large scale power by ionizing overdensities first.

Moreover, as emphasized in Paper I, the power spectrum does not uniquely determine the statistics of the $\delta T_{b}$ field. Although the density fluctuations are nearly gaussian on the relevant scales, the H II regions are not. We now consider the pixel distribution function (PDF) in the two models; i.e. the fraction of spherical "pixels" of a given mass $m_{\text {pix }}$ that have brightness temperature $\delta T_{b}$. We showed how to compute this for the inside-out model in Paper I. ${ }^{7}$ The same technique applies to the void model once we modify the excursion set barrier appropriately. ${ }^{8}$ We show some example PDFs in Figure 4 they assume $m_{\text {pix }}=10^{13} \mathrm{M}_{\odot}$ or an angular resolution $\sim 2.7^{\prime}$. The Figure explicitly demonstrates that although both reionization models increase the variance in $\delta T_{b}$, they do so in qual-

\footnotetext{
${ }^{6}$ As described in Paper I, our model probably begins to break down at this point because the internal structure of the H II regions becomes important.

${ }^{7}$ Note that we do not include overlap with large bubbles in the results shown here, so these PDFs cannot be directly compared with observations; see Paper I.

${ }^{8}$ In this case $x_{H}(\delta)$ is a monotonic function, so the resulting PDF is nonsingular.
}

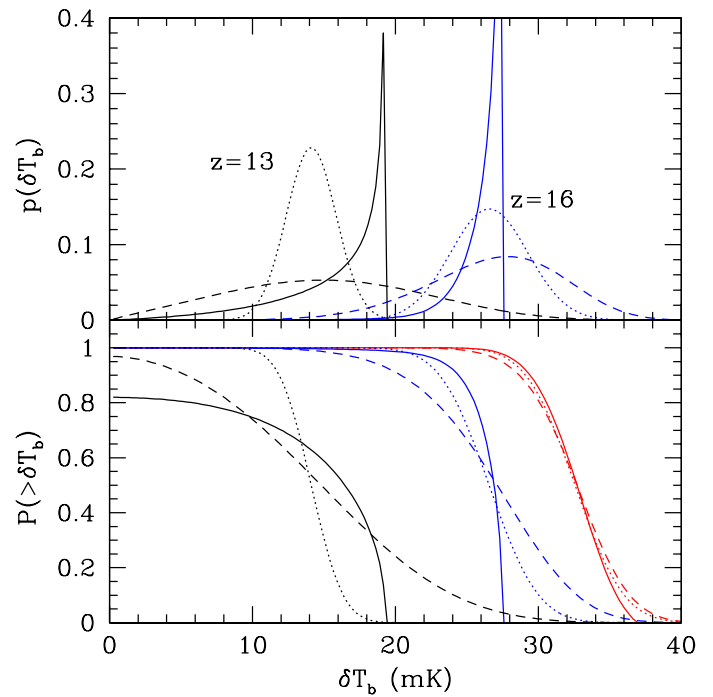

FIG. 4.- The PDF (top panel) and cumulative distribution function $P\left(>\delta T_{b}\right)$ (bottom panel) in our three models, assuming $\zeta=40$ and $m_{\text {pix }}=$ $10^{13} \mathrm{M}_{\odot}$. The solid, dashed, and dotted curves are for the inside-out model of Paper I, the outside-in void model, and a uniformly ionized IGM, respectively. In the bottom panel, the three sets of curves are for $z=20\left(\bar{x}_{H}=0.98\right)$, $z=16\left(\bar{x}_{H}=0.88\right)$ and $z=13\left(\bar{x}_{H}=0.5\right)$, from right to left. We only show the PDFs for the latter two cases.

itatively different ways. For inside-out reionization, high density regions are highly ionized and $\delta T_{b}$ has a maximum value $\delta T_{\max }$ on any given scale. The pixels tend to cluster strongly around this maximum value because $\delta T_{b}$ is a weak function of density in the relevant range; the PDF has large variance because the mean $\delta \bar{T}<\delta T_{\max }$ thanks to fully ionized regions. For outside-in reionization, on the other hand, high-density pixels remain mostly neutral, while the peak and the low density tail get stretched to small $\delta T_{b}$. The result is not so far from a gaussian, but $x_{H}(\delta)$ has dramatically increased the variance. The bottom panel shows the cumulative distribution functions $P\left(>\delta T_{b}\right)$ for three redshifts, which is often useful in statistical tests. Note that at early times, when $\bar{x}_{H}$ is small, the distributions closely resemble each other, but significant features appear even when the ionized fraction is only $\sim 10 \%$. Also, the inside-out model has $p\left(\delta T_{b}=0\right) \sim 0.2$ at $z=13$, because the characteristic bubble size is near $m_{\text {pix }}$. Figure 4 shows the necessity of identifying useful tests for non-gaussianity in the signal. We intend to explore such tests in the future.

\section{DISCUSSION}

We have examined what $21 \mathrm{~cm}$ tomography of the highredshift Universe can teach us about the ionization history. Using the reionization model described in Paper I, we predicted the angular power spectra of the brightness temperature $\delta T_{b}$ in several qualitatively different reionization scenarios. We have shown that $21 \mathrm{~cm}$ measurements offer a powerful probe of the reionization process, even without high signal-tonoise maps. Not only can they easily determine the timing of reionization, but particular ionization histories have measurable effects on the power spectrum. For example, we showed that the "bubble" feature in the power spectrum disappears if a substantial fraction of the ionizations are due to hard photons that can travel large distances from their source. Also, we contrasted the predictions of two different prescriptions for the transition between multiple generations of sources. In one, 
the transition occurs in an approximately uniformly ionized medium. In the other, the transition occurs while the original $\mathrm{H}$ II regions are still growing. We showed that these different prescriptions lead to qualitatively different power spectra, even long after the first sources have shut off, because the first generation bubbles can imprint a persistent feature on the power spectrum. This could be especially important if the first phase of reionization ends at redshifts inaccessible to the next generation radio telescopes, because of either radio frequency interference or simple noise considerations (note that the background is approximately proportional to $[1+z]^{2.7}$ for these measurements). Finally, we contrasted our "inside-out" reionization model with predictions for an "outside-in" model in which voids are ionized before dense regions. While the power spectrum of these models differ in detail, we showed that the underlying distributions of pixel brightness temperature for a given angular resolution are dramatically different. We thus suggest that more sophisticated statistical measures of the data will be able to strongly constrain the underlying model of reionization.

Of course, we have made a number of simplifying assump-

${ }^{9}$ See http://astrophysics.phys.cmu.edu/ jbp for details on PAST.

${ }^{10}$ See http://www.lofar.org for details on LOFAR.

${ }^{11}$ See http://www.skatelescope.org for details on the SKA. tions in all of these models. Most notably, we have ignored recombinations, radiative transfer, and the anisotropic distribution of sources; we discuss these issues in more detail in Paper I. Addressing these complications in a satisfactory manner will probably require numerical simulations. However, none are likely to alter our conclusions: $21 \mathrm{~cm}$ measurements contain an unprecedented wealth of information about the timing and morphology of reionization. Our simple model shows that the qualitative statistical features of the signal can be related to the source and IGM properties. The next generation of low-frequency radio telescopes, such as the Primeval Structure Telescope, ${ }^{9}$ the Low Frequency Array,${ }^{10}$ and the Square Kilometer Array, ${ }^{11}$ should be able to measure these statistical properties (ZFH04) and place strong constraints on the reionization era.

This work was supported in part by NSF grants ACI AST 99-00877, AST 00-71019, AST 0098606, and PHY 0116590 and NASA ATP grants NAG5-12140 and NAG5-13292 and by the David and Lucille Packard Foundation Fellowship for Science and Engineering.

\section{REFERENCES}

Barkana, R., \& Loeb, A. 2001, Phys. Rep., 349, 125

Becker, R. H., et al. 2001, AJ, 122, 2850

Bond, J. R., Cole, S., Efstathiou, G., \& Kaiser, N. 1991, ApJ, 379, 440

Bromm, V., Ferrara, A., Coppi, P. S., \& Larson, R. B. 2001a, MNRAS, 328, 969

Bromm, V., Kudritzki, R. P., \& Loeb, A. 2001b, ApJ, 552, 464

Cen, R. 2003, ApJ, 591, L5

Ciardi, B., \& Madau, P. 2003, ApJ, 596, 1

Ciardi, B., Stoehr, F., \& White, S. D. M. 2003, MNRAS, 343, 1101

Cooray, A., \& Sheth, R. 2002, Phys. Rep., 372, 1

Cooray, A., \& Furlanetto, S. R. 2004, ApJ, in press (astro-ph/0402239)

Di Matteo, T., Ciardi, B., \& Miniati, F. 2004, MNRAS, submitted astro-ph/0402322

Fan, X., et al. 2002, AJ, 123, 1247

Furlanetto, S. R., Sokasian, A., \& Hernquist, L. 2004a, MNRAS, 347, 187

Furlanetto, S. R., Zaldarriaga, M., \& Hernquist, L. 2004b, ApJ, submitted astro-ph/0403697, [Paper I]

Gnedin, N. Y., \& Shaver, P. A. 2003, ApJ, submitted astro-ph/0312005

Gunn, J. E., \& Peterson, B. A. 1965, ApJ, 142, 1633

Haiman, Z., \& Holder, G. P. 2003, ApJ, 595, 1

Haiman, Z., Rees, M. J., \& Loeb, A. 1997, ApJ, 476, 458

Hansen, S. H., \& Haiman, Z. 2004, ApJ, 600, 26

Hui, L., \& Haiman, Z. 2003, ApJ, 596, 9

Kogut, A., et al. 2003, ApJS, 148, 161

Kumar, A., Padmanabhan, T., \& Subramanian, K. 1995, MNRAS, 272, 544

Lacey, C., \& Cole, S. 1993, MNRAS, 262, 627

Mackey, J., Bromm, V., \& Hernquist, L. 2003, ApJ, 586, 1

Madau, P., Meiksin, A., \& Rees, M. J. 1997, ApJ, 475, 429

Madau, P., et al. 2003, ApJ, in press (astro-ph/0310223,

Miralda-Escudé, J., Haehnelt, M., \& Rees, M. J. 2000, ApJ, 530, 1

Mo, H. J., \& White, S. D. M. 1996, MNRAS, 282, 347
Morales, M. F., \& Hewitt, J. 2003, ApJ, submitted astro-ph/0312437

Oh, S. P. 2001, ApJ, 553, 499

Oh, S. P., \& Mack, K. J. 2003, MNRAS, 346, 871

Press, W. H., \& Schechter, P. 1974, ApJ, 187, 425

Ricotti, M., \& Ostriker, J. P. 2003, MNRAS, submitted (astro-ph/0311003

Sciama, D. W. 1982, MNRAS, 198, 1P

Scott, D., \& Rees, M. J. 1990, MNRAS, 247, 510

Sheth, R. K. 1998, MNRAS, 300, 1057

Sheth, R. K., \& van Weygaert, R. 2003, MNRAS, submitted astro-ph/0311260,

Sokasian, A., Abel, T., \& Hernquist, L. 2002, MNRAS, 332, 601

Sokasian, A., Abel, T., Hernquist, L., \& Springel, V. 2003a, MNRAS, 344, 607

Sokasian, A., Yoshida, N., Abel, T., Hernquist, L., \& Springel, V. 2003b, MNRAS, in press (astro-ph/0307451)

Songaila, A. 2004, AJ, in press, lastro-ph/0402347

Spergel, D. N., et al. 2003, ApJS, 148, 175

Springel, V. \& Hernquist, L. 2003, MNRAS, 339, 312

Theuns, T., et al. 2002, ApJ, 567, L103

Venkatesan, A., Giroux, M. L., \& Shull, J. M. 2001, ApJ, 563, 1

White, R. L., Becker, R. H., Fan, X., \& Strauss, M. A. 2003, AJ, 126, 1

Wyithe, J. S. B., \& Loeb, A. 2003, ApJ, 588, L69

-. 2004, Nature, 427, 815

Yoshida, N., Bromm, V., \& Hernquist, L. 2003, ApJ, in press astro-ph/0310443,

Zaldarriaga, M. 1997, Phys. Rev. D, 55, 1822

Zaldarriaga, M., Furlanetto, S. R., \& Hernquist, L. 2004, ApJ, in press (astro-ph/0311514, [ZFH04] 